\title{
MODELLING AND PI CONTROL OF AN IRRIGATION CANAL
}

\author{
X. Litrico*, V. Fromion ${ }^{\dagger}$, J.-P. Baume* ${ }^{*}$ M. Rijo ${ }^{\ddagger}$ \\ * Cemagref, UR Irrigation, B.P. 5095, 34033 Montpellier Cedex 1, France. e-mail: \\ \{xavier.litrico, jean-pierre.baume\}@ecmagref.fr \\ $\dagger$ INRA, LASB, 2 place Viala, 34060 Montpellier, France. e-mail: fromion@ensam. inra. fr \\ ¥ Universidade de Évora, Departamento de Engenharia Rural, Colégio da Mitra, Apartado 94, 7002-554 Évora, Portugal. \\ e-mail: rijo@uevora.pt
}

Keywords: Open-channel system, Saint-Venant model, PI control, Time Delay Systems, performance limitations.

\begin{abstract}
The main goal of this paper is to expose and validate a methodology to design efficient automatic controllers for irrigation canals, based on the Saint-Venant model. This model-based methodology enables to design controllers at the design stage (when the canal is not already built). The methodology is applied on an experimental canal located in Portugal. First the full nonlinear PDE model is calibrated, using a single steady-state experiment. The model is then linearized around a functioning point, in order to design linear PI controllers. Two classical control strategies are tested (local upstream control and distant downstream control) and compared on the canal. The experimental results shows the effectiveness of the method.
\end{abstract}

\section{Introduction}

The control of irrigation canals has been the subject of numerous scientific publications since the introduction of computers in the management of such large and complex systems [4]. However, only few of the proposed controllers have been effectively tested in a real situation [16]. In this paper we validate on a real system a model-based methodology to design PI automatic controllers for irrigation canals.

The problem of designing controllers for irrigation canals is difficult, because their management involves many different aspects. From a control point of view, this problem is reduced to the control of water levels at some specific points in the main canal. In this case, since the water distribution is done by gravity offtakes, a "good" distribution is obtained by maintaining a constant water level at the offtake. In order to simplify the exposition, we will consider the case where the control specification has been expressed in terms of controlling water levels upstream of control structures, and only consider classical control design methods (PI controllers). This is a first step validation for more advanced control design methods $[12,10]$ that will be validated in another paper [11].

From a control point of view, we want to control a nonlinear system around equilibrium points with a linear controller (typically a PI). This requires a linear model of the system around the given equilibrium points.
Two main approaches can be followed to obtain a linear model in the case of irrigation canals:

1. To use identification tools [17],

2. To linearize a knowledge-based full nonlinear model, i.e. Saint-Venant equations and hydraulic structures equations (see [13] and references therein).

The cost of each approach in terms of experimental data can be summarized as follows:

1. The first approach requires to use the canal to identify the dynamics. Experiments have to cover all the required setpoints of the system: a set of discharge values between maximum and minimum values, set of downstream limit conditions, set of gate openings. This has to be done on each pool of the canal, which leads to a large number of experiments, and model identifications. The system is thus represented by a large number of linear systems.

2. The second approach necessitates the data needed to calibrate a full Saint-Venant model for simulation purposes. Actually, one needs the canal geometry, and a single experiment in steady state (for the canal or for each pool), in order to calibrate Manning coefficients and gate discharge coefficients.

At this stage, it should be clear that the second approach is less demanding in terms of data, since the geometry is usually available in most of the existing canals. Moreover, a good estimate of Manning coefficient can usually be deduced from the canal material -this is the basis of the (structural) design of irrigation canals- and even the gate discharge coefficients can be estimated from the structure geometry [8]. Therefore, once the geometry is known, this knowledge-based model can easily be obtained. This enables to have an integrated design method for irrigation canals: the automatic control scheme can be integrated into the structural design of irrigation canals.

Since the objective is to design linear controllers, one needs linear models. For this purpose, let us examine the implications of both approaches:

1. In the first approach, the linear models obtained from identification can be used directly. A difficulty that arises is: How to quantify the uncertainty? 
2. For the second approach, linear models have to be deduced from Saint-Venant equations. The dynamic uncertainty is then directly linked to the physical parameters uncertainties (e.g. uncertainty on coefficients of SaintVenant equations, actuator uncertainty, sensor uncertainty, etc.).

If the full nonlinear model is valid, then the second approach encompasses the first one. In fact, a linear model could be directly identified on the nonlinear simulator (see [7], [6]).

However, even if Saint-Venant equations have been used by hydraulic engineers for simulation purposes, the obtention of linear models for controller design directly from the equations needs to be clarified. A first way is to consider only uniform regimes (where discharge and water depth are constant along the pool) and the associated analytical solution in the Laplace domain (see [2] [3]). In this case, how to describe the behavior in non uniform cases (i.e. the vast majority of cases)? A tentative step to approximate the transfer function has been done by [16], but did not lead to an exact solution.

A second way is to use numerical schemes (e.g. Preissmann scheme) to deduce linear models (see [13], [1], [5]). In this case, how the obtained approximate model can be validated from a control point of view (in contrast to the classical validation in the time-domain)?

In conclusion of this short discussion, the use of a model-based method has been largely undermined by the difficulties linked to both ways. Recent works allow to bypass these difficulties, by the obtention of a continuous linear model directly from Saint-Venant equations for any regime [9].

On this basis, we propose a complete model-based methodology to design controllers for irrigation canals. This methodology can be summarized in four successive steps:

1. Obtain the data necessary for the full nonlinear model: canal geometry, hydraulic structures description, steady state measurements (upstream discharge, upstream and downstream water levels at each structure, gate openings).

2. Calibrate the model:

- hydraulic model calibration: determine Manning and discharge coefficients using the steady state measurements,

- dynamic model calibration: model the actuators, sensors and data transmission dynamics, using either the data given the constructor, either direct identification on step response.

3. Obtain linear models based on the calibrated full nonlinear one.

4. Design controllers using the linear models. In a first approximation, the actuator/sensors dynamics can be neglected, since they are usually very rapid compared to the canal dynamics.
The methodology is exposed and applied for simple controllers (PI). This allows to use classical design methods in order to validate the general methodology, by applying it to a real system. Once the linear models are validated, it is easy to use them to design more sophisticated controllers. Such an approach is developed in another paper [11], which examines the performance requirements attached to the control of irrigation canals.

The paper is structured as follows: a brief description of the experimental canal is firstly given, then the methodology is exposed, and applied to this specific case. Experimental results validating the approach are finally presented and discussed.

\section{Canal description}

The automatic canal used in the present study is a component of the experimental facility of the Hydraulics and Canal Control Center (NuHCC) of the University of Évora (Portugal).

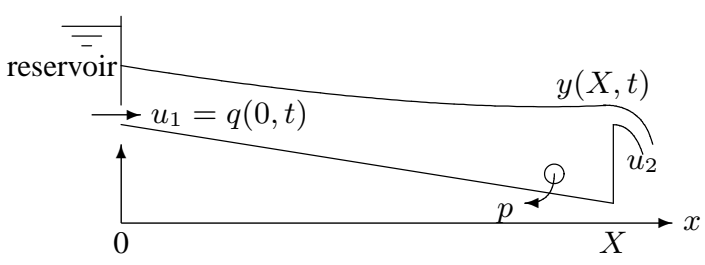

Figure 1: Schematic representation of the experimental canal

Design The experimental canal is a trapezoidal and lined canal, with a general cross section of bottom width $0.15 \mathrm{~m}$, sides slope 1:0.15 (V:H) and depth $0.90 \mathrm{~m}$. The last $7 \mathrm{~m}$ downstream of canal have a rectangular cross section of $0.7 \mathrm{~m}$ width. The overall canal is $145.5 \mathrm{~m}$ long and the average longitudinal bottom slope is about $1.5 \times 10^{-3}$. The design flow is 0.09 $\mathrm{m}^{3} \mathrm{~s}^{-1}$.

A longitudinal view of the canal is schematized in figure 1.

Control devices The canal inlet is equipped with a motorized flow control valve, that delivers a discharge $u_{1}$. The downstream end is controlled with a rectangular sluice gate $u_{2}$ (overshot gate).

Outlet An offtake $p$ is located at the downstream end of the pool, equipped with an electromagnetic flowmeter and a motorized butterfly valve.

Sensors A water level sensor is installed within an offline stilling well at the downstream end of the pool, measuring the downstream water depth $y$. The sensor is of float and counterweight type attached by a stainless steel tape; this tape runs over a sprocket wheel. The wheel movements are transmitted to a potentiometer that transmits to the controller the analogical input corresponding to the water surface. 


\section{Modelling of an irrigation canal}

\subsection{Hydraulic model}

The Saint-Venant equations are used to model the flow in the canal. These equations are nonlinear hyperbolic partial differential equations involving the average discharge $Q(x, t)$ and the water depth $Y(x, t)$ along one space dimension $x$ [4]:

$$
\begin{aligned}
\frac{\partial A}{\partial t}+\frac{\partial Q}{\partial x} & =0 \\
\frac{\partial Q}{\partial t}+\frac{\partial Q^{2} / A}{\partial x}+g A \frac{\partial Y}{\partial x} & =g A\left(I-S_{f}\right)
\end{aligned}
$$

with $A(x, t)$ is the wetted area $\left[\mathrm{m}^{2}\right], Q(x, t)$ the discharge $\left[\mathrm{m}^{3} / \mathrm{s}\right]$ across section $A, Y(x, t)$ the water depth [m], $S_{f}(x, t)$ the friction slope, $I$ the bed slope and $g$ the gravitational acceleration $\left[\mathrm{m} / \mathrm{s}^{2}\right]$.

Two boundary conditions are necessary for this partial differential system, for example $Q(0, t)=Q_{0}(t)$ and $Q(X, t)=$ $Q_{X}(t)$, where $X$ is the length of the considered channel. The initial conditions are given by $Q(x, 0)$ and $Y(x, 0)$.

The friction slope $S_{f}$ is modelled with Manning-Strickler formula:

$$
S_{f}=\frac{Q^{2} n^{2}}{A^{2} R^{4 / 3}}
$$

with $n$ the Manning coefficient $\left[\mathrm{sm}^{-1 / 3}\right]$ and $R$ the hydraulic radius [m], defined by $R=A / P$, where $P$ is the wetted perimeter $[\mathrm{m}]$.

\section{Hydraulic structure equation}

The hydraulic structure (over shot gate) is modelled using the classical equation (free flow case):

$$
Q=C_{d} L_{v} \sqrt{2 g}(Y-W)^{3 / 2}
$$

with $Q$ the discharge through the structure, $Y$ the upstream water level, $W$ the sill elevation and $L_{v}$ the gate width.

\subsubsection{Steady flow model calibration}

For a given constant upstream discharge ( $45 \mathrm{l} / \mathrm{s})$, the water level and sill elevation were monitored. This steady flow period enabled to identify the Strickler coefficient $K$ for each pool and the discharge coefficient at each structure. The calibration lead to a discharge coefficient of 0.4 for the overshot gate and a Strickler coefficient of 60 for the canal.

\subsubsection{Unsteady flow model validation}

The nonlinear model calibrated in steady flow is validated in unsteady flow for different flow configurations. For simulation purposes, we used SIC, a computer program developed by Cemagref [14]. This mathematical model solves the full nonlinear Saint-Venant equations using a finite difference scheme (Preissmann scheme). Figure 2 corresponds to the same regime
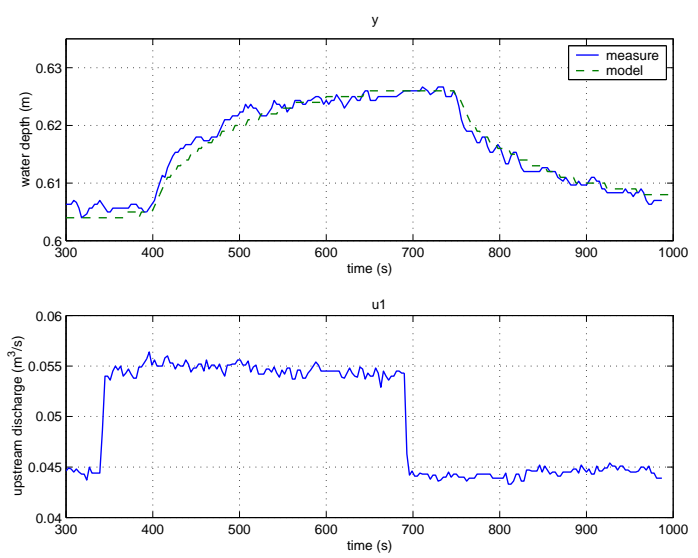

Figure 2: Step response around $Q_{0}=45 \mathrm{l} / \mathrm{s}$, downstream boundary condition $y_{0}=0.6 \mathrm{~m}$

as the one used for the steady flow calibration. The model fits very well the data.

In figure 3, the downstream boundary condition has changed. The model is still able to accurately reproduce the level variations.
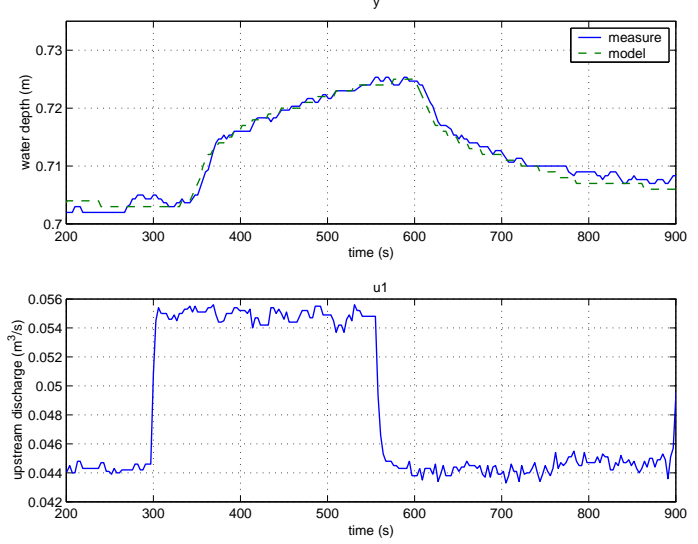

Figure 3: Step response around $Q_{0}=45 \mathrm{l} / \mathrm{s}$, downstream boundary condition $y_{0}=0.7 \mathrm{~m}$

Many other simulations were done to validate the model, which appeared to be very accurate, even for different flow conditions. This validates the Saint-Venant model for this canal.

\subsection{Control oriented model}

\subsubsection{Linear hydraulic model}

The linear model used for control design is obtained following [9]: we consider small variations of water depth $y(x, t)$ and discharge $q(x, t)$ around stationary values defined by $Y_{0}(x)(\mathrm{m})$ and $Q_{0}(x)\left(\mathrm{m}^{3} / \mathrm{s}\right)$. This leads to the linearized Saint-Venant 
equations:

$$
\begin{aligned}
L_{0} \frac{\partial y}{\partial t}+\frac{\partial q}{\partial x} & =0 \\
\frac{\partial q}{\partial t}+2 V_{0} \frac{\partial q}{\partial x}-\beta_{0} q+\left(C_{0}^{2}-V_{0}^{2}\right) L_{0} \frac{\partial y}{\partial x}-\gamma_{0} y & =0
\end{aligned}
$$

$L_{0}$ is the top width for the equilibrium regime (m), $V_{0}$ the average velocity $(\mathrm{m} / \mathrm{s})$ and $C_{0}=\sqrt{g A_{0} / L_{0}}$ the wave celerity $(\mathrm{m} / \mathrm{s})$. Moreover, one has $\gamma_{0}=$ $V_{0}^{2} \frac{\partial L_{0}}{\partial x}+g L_{0}\left[(1+\kappa) I-\left(1+\kappa-F_{0}^{2}(\kappa-2)\right) \frac{\partial Y_{0}}{\partial x}\right], \beta_{0}=$ $-\frac{2 g}{V_{0}}\left(I-\frac{\partial Y_{0}}{\partial x}\right)$ and $\kappa=\frac{7}{3}-\frac{4 S_{0}}{3 L_{0} P_{0}} \frac{\partial P_{0}}{\partial Y} . \quad F_{0}$ is the Froude number $F_{0}=\frac{V_{0}}{C_{0}}$. A transfer matrix linear model that can be used for control purposes is obtained using Laplace transform and a specific numerical method [9].

As a linearized model can be obtained from the computer program SIC, we compare the continuous time linear model with the discrete time model obtained with the finite difference scheme [13] (see figure 4). It is clear that the frequency domain responses of the models are very close up to a decade below the Nyquist frequency.

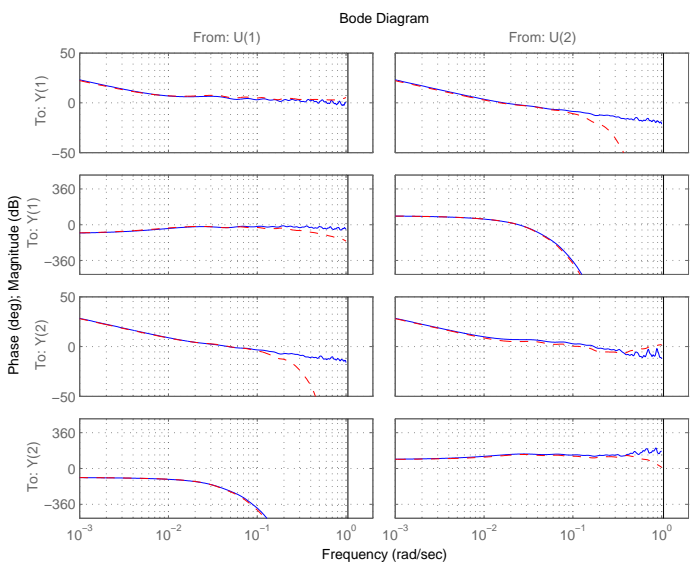

Figure 4: Bode plots of the continuous model (-) and of the finite difference model (- $)$, for $Q=0.045 \mathrm{~m}^{3} / \mathrm{s}, Y_{X}=0.6$ $\mathrm{m}$.

This model needs to be completed in our case with a model of the actuators and sensors dynamics. The equations describing hydraulic structures interactions with the flow are linearized and added to the model. The hydraulic structure (overshot gate) is modelled using the linearized equation:

$$
q(X, t)=k_{1} y(X, t)+k_{2} u_{2}(t)
$$

with $q(X, t)$ the discharge through the structure, $y(X, t)$ the upstream water level, $u_{2}$ the gate opening. Coefficients $k_{1}, k_{2}$ are obtained by linearizing the structure equation (4) around a given functioning point.

\subsubsection{Actuators and sensors modelling}

Actuators and sensors dynamics are identified using their step responses. They are represented by linear models of first order with delay. The upstream actuator $u_{1}$ delivering a discharge is modelled with the following transfer function:

$$
f_{1}(s)=\frac{e^{-4 s}}{8 s+1}
$$

The downstream actuator (overshot gate) $u_{2}$ is modelled with the following transfer function:

$$
f_{2}(s)=0.415 \frac{e^{-2 s}}{3 s+1}
$$

Putting together these transfer functions with the linear model obtained from the hydraulic nonlinear PDE model (SaintVenant equations), leads to the model for controller design:

$$
y=G_{1}(s) u_{1}+G_{2}(s) u_{2}+\tilde{G}(s) p
$$

where $y$ is the downstream water level, $u_{1}$ the upstream control variable (a discharge), $u_{2}$ the downstream control variable (gate opening), and $p$ is a perturbation corresponding to the outlet flow.

This model will be used to design simple monovariable PI controllers.

\section{PI controllers design}

\subsection{Control politics for an irrigation canal}

The real-time management of an open-channel irrigation canal is a difficult task, especially because of the time-lag between the water resource (located upstream) and the water user (located downstream). In fact, a simple way to satisfy water needs would be to deliver a constant upstream discharge corresponding to the maximal water demand, and to let the remaining discharge flow downstream. This type of management corresponds to the so-called upstream control strategy, used in the majority of irrigated perimeters managed with a scheduled rotational delivery.

An opposite way to manage the system would be to deliver only the necessary water requested by the users (downstream control strategy). Then, since a time-delay is necessary to transport water from the reservoir to the user, this control strategy cannot immediately satisfy the water demands.

Taken into account the physical limitations in terms of civil engineering, the two classical ways to control an irrigation canal are as follows [15]:

- distant downstream control, where the upstream control variable $u_{1}$ is manipulated to control a water level $y$ located at the downstream end of the pool,

- local upstream control, where the downstream control variable $u_{2}$ is manipulated to control a water level $y$ located just upstream.

In the following, PI controllers will be designed for each of these classical control strategies, using the obtained model. 


\subsection{Distant downstream control}

The real-time performance of distant downstream control is limited by the time-delay between upstream discharge and downstream level (here $\tau=60 \mathrm{~s}$ ). The time-delay structurally limits the achievable bandwidth up to about $1 / \tau$ (see [12]). The tuning of the controller is done using classical rules in order to get the desired gain and phase margins (in our case a gain margin of $8 \mathrm{~dB}$ and at least 60 degrees of phase margin, see figure $5)$.

The distant downstream controller is obtained as:

$$
K_{1}(s)=\frac{0.455 s+4.5 \times 10^{-3}}{s}
$$

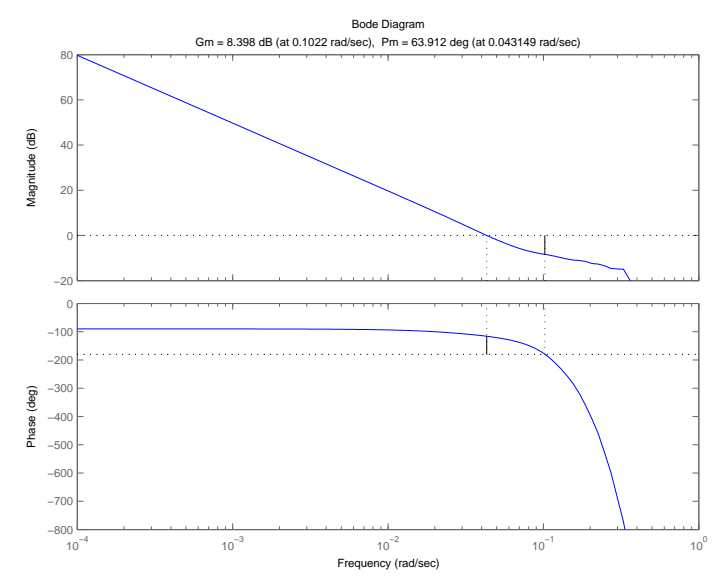

Figure 5: Bode plot of $K_{1} G_{1}$ for the distant downstream controller

Figure 6 gives the experimental results obtained with the distant downstream PI controller. A downstream withdrawal of 10 $1 / \mathrm{s}\left(0.01 \mathrm{~m}^{3} / \mathrm{s}\right)$ is done at time $t=280 \mathrm{~s}$. The controller is able to reject this unknown perturbation in about $400 \mathrm{~s}$ (time for the output $y$ to meet the target $y_{c}=0.6 \mathrm{~m}$ ). The outlet is closed at time $t=750 \mathrm{~s}$, and the response appears to be symmetrical.

\subsection{Local upstream control}

Local upstream control is not subject to control performance limitations, since there is no delay between the actuator and the controlled variable (in fact, it can be shown that the transfer function is outer). However, the actuator dynamics induce physical limitation on the control.

The PI controller is tuned in order to have a gain margin of $12 \mathrm{~dB}$ (in order not to control the first resonant mode of the system) and at least 60 degrees of phase margin, see figure 7 .

The local upstream controller is given by:

$$
K_{2}(s)=\frac{2 s+0.1}{s}
$$

Figure 8 gives the experimental results obtained with the local upstream PI controller. The simulation with a linear model is
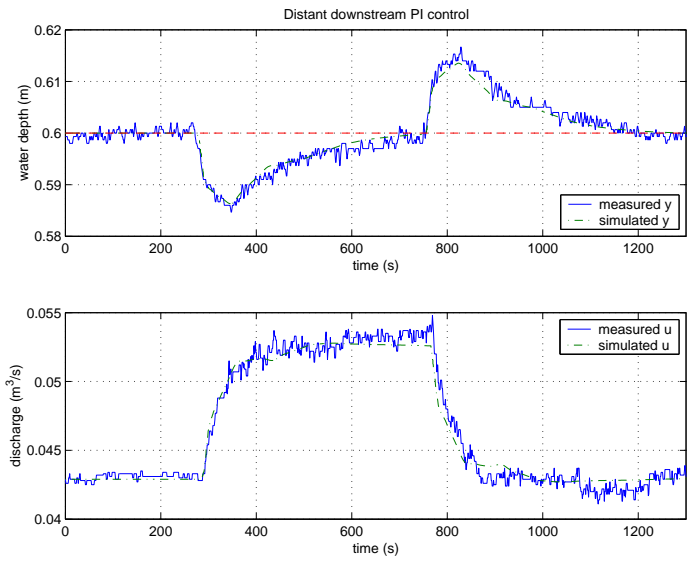

Figure 6: Experimental response of the distant downstream PI controller to a downstream withdrawal, comparison with a linear simulation

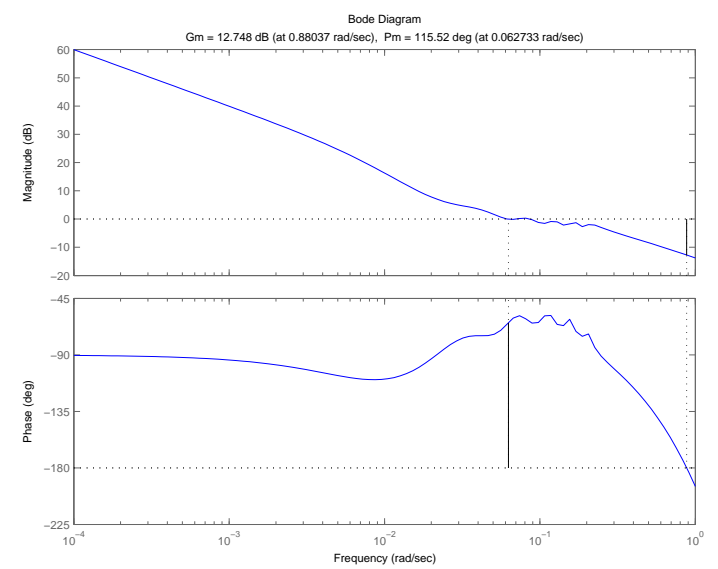

Figure 7: Bode plot of $K_{2} G_{2}$ for the local upstream controller

able to rather accurately reproduce the dynamic behavior of the system in response to a downstream withdrawal.

A downstream withdrawal of $101 / \mathrm{s}\left(0.01 \mathrm{~m}^{3} / \mathrm{s}\right)$ is done at time $t=120 \mathrm{~s}$. The controller is able to reject this unknown perturbation in about $100 \mathrm{~s}$, which is about 4 times quicker than with the downstream controller.

In the local upstream control case, the bandwidth is significantly higher than in the distant downstream control case. The measurement noise is then amplified by the control system. This explains the difference between the linear simulated control and the measured one in figure 8 (there is a dead band of 5 $\mathrm{mm}$ on the control action $u_{2}$ ). The measurement noise should be filtered in order to have a better fit. In fact, part of the gain margin is used to take this into account. 

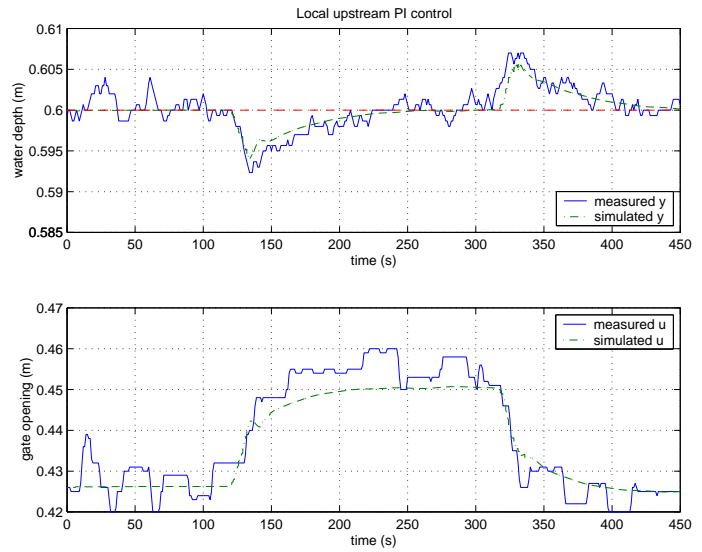

Figure 8: Experimental response of the local upstream PI controller to a downstream withdrawal, comparison with a linear simulation

\section{Conclusion}

The paper has exposed and validated a methodology to design efficient automatic controllers for irrigation canals, based on the Saint-Venant model. The methodology has been applied on an experimental canal located in Portugal. The interest of the method is to greatly simplify the model calibration (a single experiment is needed to calibrate the model in steady state). Linear models around different reference points can then be obtained using recent results [9].

Two types of PI controllers were designed and field tested:

- a distant downstream PI controller, where the downstream water elevation $y$ is controlled using the upstream discharge $u_{1}$,

- a local upstream PI controller, where the downstream water level $y$ is controlled using the downstream gate $u_{2}$.

The experimental results are very satisfactory for classical PI controllers design for local upstream and distant downstream control. The proposed model-based methodology is therefore validated for PI controllers; these promising results will be generalized to the control of multiple canal pools.

\section{Acknowledgments}

This work was supported by the French Embassy in Portugal and GRICES (Gabinete de Relações Internacionais da Ciência e do Ensino Superior) of Portugal, through the scientific collaboration project $\mathrm{n}^{\circ}$ 547-B4 : "Modélisation hydraulique et tests de régulateurs automatiques pour le canal réduit d'Évora”.

The first two authors acknowledge the financial help of Cemagref and INRA through the collaborative program ASS AQUAE $\mathrm{n}^{\circ} 02$ on the control of delayed hydraulic systems.

\section{References}

[1] O.S. Balogun. Design of real-time feedback control for canal systems using linear quadratic regulator theory. Ph.D. thesis, Department of Mechanical Engineering, University of California at Davis, 1985. $230 \mathrm{pp}$.

[2] J.P. Baume and J. Sau. Study of irrigation canal dynamics for control purposes. In Int. Workshop RIC'97, pages 3-12, Marrakech, Morroco, 1997.

[3] G. Corriga, F. Patta, S. Sanna, and G. Usai. A mathematical model for open-channel networks. Applied Mathematical Modelling, 3:51-54, 1979.

[4] J.A. Cunge, F.M. Holly, and A. Verwey. Practical aspects of computational river hydraulics. Pitman Advanced Publishing Program, 1980.

[5] A. Garcia. Control and regulation of open channel flow. M.Sc. thesis, University of California, Davis, 1988.

[6] H. Jreij. Sur la régulation des cours d'eau aménagés. Ph.D. thesis, Université Paris-XI Dauphine, 1997. (in French).

[7] P. Kosuth. Techniques de régulation automatique des systèmes complexes : application aux systèmes hydrauliques à surface libre. Ph.D. thesis, Institut National Polytechnique de Toulouse, 1994. (in French).

[8] A. Lencastre. Hydraulique générale. Eyrolles, SAFEGE, 1996.

[9] X. Litrico and V. Fromion. Infinite dimensional modelling of open-channel hydraulic systems for control purposes. In $41^{\text {st }}$ Conf. on Decision and Control, pages 1681-1686, Las Vegas, 2002.

[10] X. Litrico and V. Fromion. Real-time management of multireservoir hydraulic systems using $H_{\infty}$ optimization. In IFAC World Congress, Barcelona, 2002.

[11] X. Litrico and V. Fromion. Advanced control politics and optimal performance for an irrigation canal. In European Control Conference, Cambridge, UK, 2003.

[12] X. Litrico, V. Fromion, and G. Scorletti. Improved performance for open-channel hydraulic systems using intermediate measurements. In IFAC Workshop on Time-Delay Systems, pages 113118, Santa Fe, 2001.

[13] P.-O. Malaterre. Modélisation, analyse et commande optimale LQR d'un canal d'irrigation. Ph.D. thesis, ISBN 2-85362-3688, Etude EEE ${ }^{\circ}$. 14, LAAS - CNRS - ENGREF - Cemagref, 1994. (in French).

[14] P.-O. Malaterre and J.-P. Baume. Sic 3.0, a simulation model for canal automation design. In Int. Workshop on the Regulation of Irrigation Canals RIC'97, Marrakech (Morocco), April 22-24 1997.

[15] P.-O. Malaterre, D. C. Rogers, and J. Schuurmans. Classification of canal control algorithms. J. of Irrigation and Drainage Engineering, 124(1):3-10, January/February 1998. ISSN 07339437.

[16] J. Schuurmans. Control of water levels in open-channels. Ph.D. thesis, ISBN 90-9010995-1, Delft University of Technology, 1997.

[17] E. Weyer. System identification of an open water channel. Control Engineering Practice, 9:1289-1299, 2001. 